\title{
Fiabilidad técnica del bloqueo caudal guiado con ecografía
}

\author{
A. Mendiola de la Osa ${ }^{1}$, J. Insausti Valdivia ${ }^{1}$, M. Martín Ayuso $^{2}$, E. Pellejero Collado ${ }^{3}$ \\ y J.M. Valverde Mantecón² \\ ${ }^{1}$ Unidad del Dolor. Servicio Anestesiología, Reanimación y Terapéutica del Dolor. ${ }^{2}$ Servicio Anestesiología, \\ Reanimación y Terapéutica del Dolor. ${ }^{3}$ Unidad del Dolor. Hospital General de Ciudad Real. Ciudad Real
}

Mendiola de la Osa A, Insausti Valdivia J, Martín Ayuso M, Pellejero Collado E, Valverde Mantecón JM. Fiabilidad técnica del bloqueo caudal guiado con ecografía. Rev Soc Esp Dolor 2014; 21(6): 323-327.

\begin{abstract}
Introduction: Caudal block is a procedure used to treat lumbo-sacral root pain. This procedure has traditionally been realized using anatomy references or guided by fluoroscopy. The realization of sonography-guided technique and complications following the embodiment there of is presented

Material and methods: Observational and descriptive study in which is studied procedure complications. One hundred and twenty-six caudal blocks were performed in adult patients of both sexes.

Procedure: The sacral hiatus was located by ultrasound and 18 G Tuohy needle was inserted into the epidural space flow drilling the sacro-coccygeal ligament.

Results: There were no serious complications arose in any of the patients. There were no cases of poor ultrasound window so they could perform the technique on all scheduled patients.

Conclusions: The procedure sonography-guided is safe and reliable due to the absence of complications and is an alternative to flouoroscopy-guided technique. This technique of ionizing radiation exposure is avoided. In addition the caudal block does not need ionized contrast substances and let take the sonography machine bedsides if necessary.
\end{abstract}

Key words: Caudal block. Sonography.

\section{RESUMEN}

Introducción: el bloqueo-infiltración caudal es una técnica utilizada en las unidades de dolor para tratar el dolor de origen radicular lumbosacro. Esta técnica se ha realizado clásicamente mediante referencias anatómicas o guiado mediante fluoroscopia. Se presenta la realización de la técnica eco-guiada y las complicaciones surgidas tras la realización de la misma.

Material y métodos: estudio observacional descriptivo en el que se estudiaron las complicaciones de la técnica. Se realizaron 126 procedimientos en pacientes adultos de ambos sexos.

Procedimiento: se localizó el hiato sacro mediante ecografía y se insertó una aguja de Touhy $18 \mathrm{G}$ en el espacio epidural caudal perforando el ligamento sacro-coccígeo.

Resultados: no surgieron complicaciones graves en ninguno de los pacientes. No hubo casos de mala ventana ecográfica por lo que se pudo realizar la técnica en todos los enfermos programados.

Conclusión: debido a la ausencia de complicaciones la realización de la técnica eco-guiada es una alternativa segura y fiable a la técnica guiada con fluoroscopia. Mediante esta técnica se evita la exposición de radiaciones ionizantes. Además el bloqueo caudal eco-guiado no necesita del uso de contrastes yodados y permite transportar el ecógrafo a la cabecera del enfermo en caso de ser necesario.

Palabras clave: Bloqueo caudal. Ecografía.

\section{INTRODUCCIÓN}

Una de las alternativas al tratamiento del dolor radicular lumbosacro (DRLS) es la administración de corticoides en el espacio epidural caudal a través del hiato sacro, técnica conocida como bloqueo caudal (1). Esta técnica tiene una eficacia cuyo nivel de evidencia es fuerte para el tratamien- 
to de DRLS a corto plazo ( $<6$ semanas), y moderado a largo plazo ( $>6$ semanas) $(1,2)$.

Clásicamente el bloqueo caudal se ha realizado mediante referencias anatómicas, fundamentalmente para realizar anestesia infantil (3). A pesar de que el hiato se encuentra a escasa profundidad, la punción a ciegas no siempre es factible debido a las variaciones anatómicas interpersonales (irregularidades óseas, formas de hiato y defectos de la pared dorsal del conducto sacro), por lo que una de las causas frecuentes del fracaso de la efectividad de la técnica es la inadecuada colocación de la punta de la aguja (4). Por este motivo se aconseja realizar el bloqueo caudal guiado mediante una técnica de imagen (5).

En las unidades de dolor, el bloqueo caudal se realiza guiado mediante fluoroscopia $(6,7)$ en la mayoría de las ocasiones, aunque también se ha descrito la técnica guiada mediante TC (8) y/o ecografía (9-11). A pesar de que el mayor inconveniente de la realización de la técnica guiada mediante fluoroscopia es la exposición a radiaciones ionizantes, tanto del personal sanitario como del paciente (12), es importante señalar las dificultades existentes para acceder a una sala de realización de técnicas guiadas mediante aparatos emisores de radiaciones ionizantes debido a las infraestructuras y medidas de seguridad necesarias (mediciones de radiación de lugares y personal en el que se realiza la técnica, pared plomada, mandiles y gafas de plomo para protección del personal sanitario y del enfermo y la presencia de personal especializado y entrenado en el manejo de estos aparatos) (13).

La realización del bloqueo caudal guiado con ecografía ha sido descrita por varios autores $(14,15)$ básicamente en niños $(16,17)$. El uso de esta técnica de imagen hace que el bloqueo caudal sea más accesible al no precisar una sala especialmente preparada ni tener que requerir la presencia de personal específico, aunque precise una familiarización con la imagen ecográfica por parte de médico.

Por lo descrito anteriormente encontramos de interés la descripción de la realización de la técnica en adultos y el estudio de las complicaciones que surgieron en nuestra unidad al llevarla a cabo.

\section{MATERIAL Y MÉTODOS}

Se realizó un estudio observacional descriptivo. Se estudiaron las complicaciones surgidas en los pacientes a los que se les realizó el bloqueo caudal eco-guiado durante los meses de junio a diciembre de 2012.

Criterios de inclusión: pacientes mayores de edad con DRLS. Se obtuvo el consentimiento informado de todos los enfermos para la realización de la técnica con más de 24 h de antelación y la autorización para la participación en este estudio. En aquellos que estaban tomando antiagregantes o anticoagulantes se siguieron las recomenda- ciones sobre la realización de técnicas anestésicas neuroaxiales $(18,19)$.

\section{REALIZACIÓN DE LA TÉCNICA}

Se utilizó la sonda lineal configurada con una frecuencia de $12 \mathrm{Mhz}$ y una profundidad de $5 \mathrm{~cm}$ de un ecógrafo Nemio 20, (Toshiba Medical Systems Corporation, Japón). Tras comprobar que los parámetros de coagulación estaban dentro de los límites normales $(18,19)$ los pacientes se colocaron en la posición de Kraske. Se monitorizó la frecuencia cardiaca y la onda de pulsioximetría (Monitor Phillips, Sure Signs VM6). Se adoptaron las medidas de esterilidad habituales para realizar una punción neuroaxial, se protegió la sonda ecográfica con una funda estéril y se utilizó un gel estéril.

En la transición lumbosacra, se colocó la sonda en eje transversal y se deslizó caudalmente hasta la visualización de los cornetes sacros junto con el hiato sacro (Fig. 1A); después se giró la sonda obteniendo un corte longitudinal observándose el hiato sacro y el ligamiento sacro-coccígeo (9). En este corte también se observó la pared dorsal del sacro y el cóccix (Fig. 1B). Se infiltró el punto de punción con lidocaína al $1 \%$ y tras esperar 1 minuto se introdujo una aguja de Touhy $18 \mathrm{G}$ "en plano" viendo como la aguja perforaba el ligamento sacro-coccígeo (Fig. 2). Una vez perforado el ligamento se perdió la visión de la parte distal de la aguja debido a la sombra ecográfica posterior generada por la pared dorsal del sacro. La aguja no se debe introducir más de $1 \mathrm{~cm}$ debido a que el saco dural termina aproximadamente a nivel de S2 (11). Tras aspirar y comprobar que la aguja no se introdujo en el plexo venoso sacro, y siempre viendo la parte proximal de la aguja, se instiló $1 \mathrm{~cm}$ de suero fisiológico rápidamente para confirmar que la punta de la aguja estaba situada en el espacio epidural sacro. Esta maniobra no debe generar ninguna modificación en la imagen debido a que la sombra posterior de la pared dorsal lo impide. Una vez confirmada la posición de la aguja se administró la medicación $(40 \mathrm{mg}$ de triamcinolona $+2 \mathrm{ml}$ de L-bupivacaína al 0,25\%+5 ml de suero fisiológico).

\section{RESULTADOS}

Durante los meses de junio a diciembre del año 2012 se realizaron en nuestra unidad 126 procedimientos en 85 pacientes, de los cuales $53(62,35 \%)$ fueron en mujeres y $32(37,64 \%)$ en varones. La edad media de los sujetos estudiados fue de 55,78 años. La técnica se pudo realizar en todos los pacientes programados no teniendo que suspenderse en ninguno por dificultades técnicas o mala ventana ecográfica. 

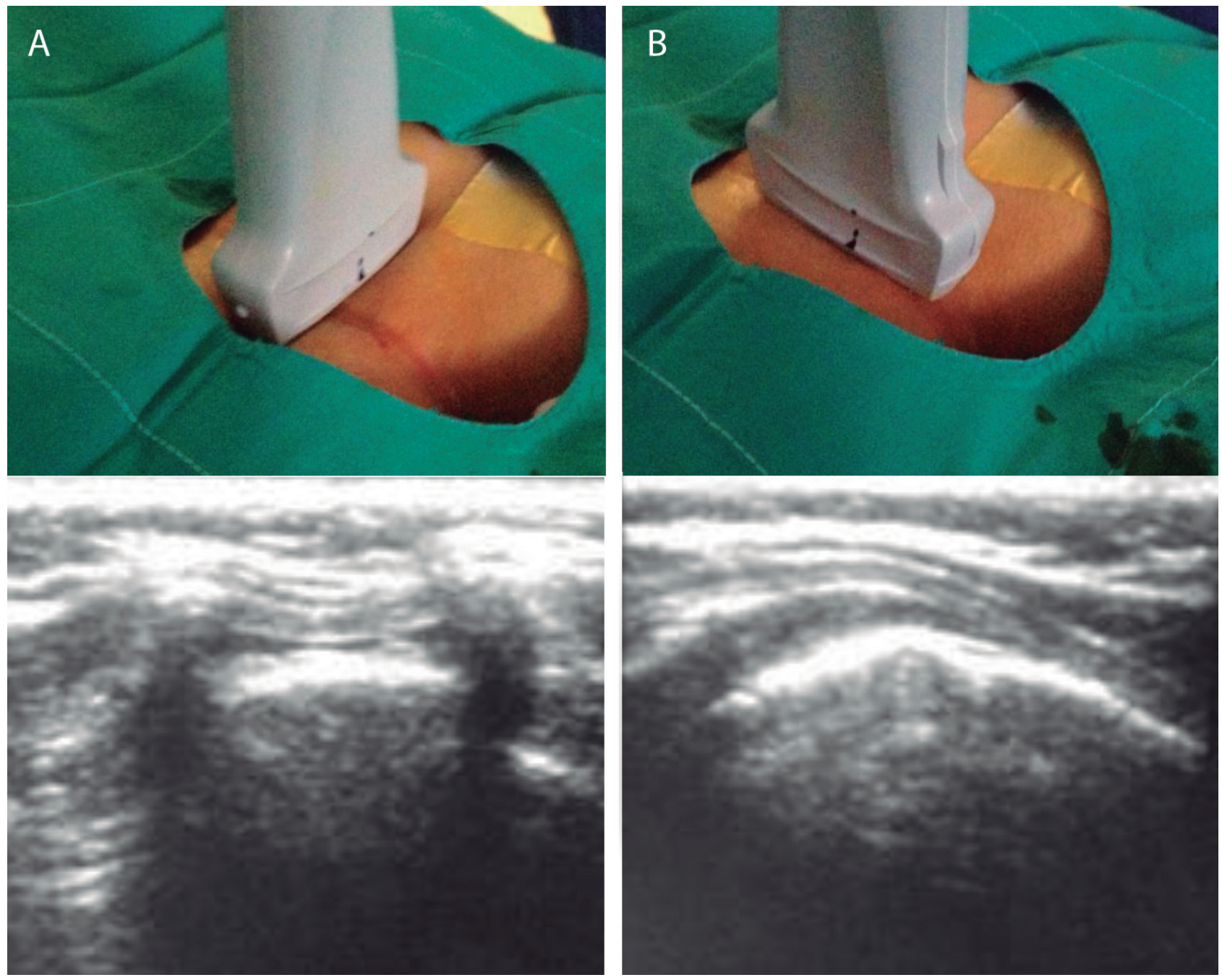

Fig. 1. Colocación de la sonda ecográfica e imagen obtenida en A: eje transversal y B: eje longitudinal.

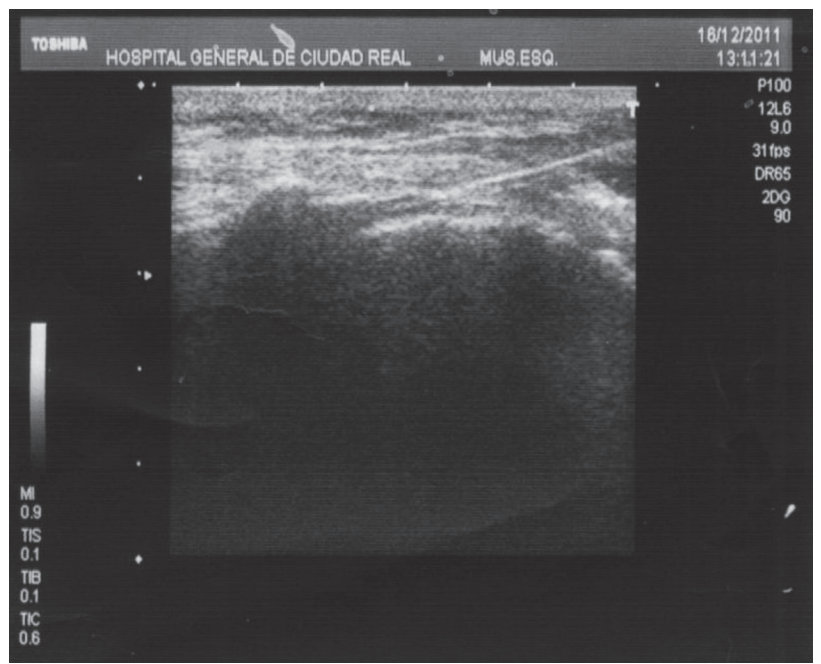

Fig. 2. Imagen ecográfica en la que se observa la aguja intoduciéndose en el hiato sacro.
En ningún caso se observaron complicaciones relacionadas con la técnica: producción de hematoma epidural con compromiso radicular, bloqueo anestésico total o efectos secundarios a la administración de anestésico local intravenoso. Un $4 \%$ sufrió una disminución de frecuencia cardiaca con sensación de mareo y náuseas (sin vómitos) que cedieron totalmente tras colocación del paciente en posición de Trendelenburg. No hubo la necesidad de administrar bolos de coloides ni cristaloides intravenosos ni de usar drogas vasoactivas. No se observaron síntomas de administración intravenosa de anestésicos locales ni de punción del plexo venoso sacro en ningún caso.

\section{DISCUSIÓN}

Los datos expuestos anteriormente avalan la realización del bloqueo caudal guiado mediante ecografía como alter- 
Rev. Soc. Esp. del Dolor, Vol. 21, N. ${ }^{\circ}$ 6, Noviembre-Diciembre 2014

nativa a la fluoroscopia. Se trata de un procedimiento seguro y fiable. Además se pone de manifiesto la ausencia de complicaciones graves aunque la realización de la técnica no está exenta de ellas (20).

Según diferentes estudios la eficacia de la técnica para el tratamiento del DRLS a largo plazo es controvertida, teniendo un nivel de evidencia moderado $(2,21)$. Sin embargo, en la realización del bloqueo caudal mediante referencias anatómicas por médicos experimentados la malposición de la aguja ocurre en más de un $25 \%(9,20)$, por lo que en este porcentaje de pacientes el bloqueo es claramente ineficaz. Por otro lado existen estudios en los que la realización del bloqueo caudal guiado mediante imagen disminuye la intensidad del DRLS tanto a corto como a largo plazo $(22,23)$.

La dosis media de radiación recibida cada vez que se realiza un bloqueo epidural vía interlaminar por el médico en la mano dominante es de 0,7 mREM y de 0,4 mREM en las gafas de protección (12). Sin embargo, en una encuesta realizada a las unidades de dolor de nuestro país se pone de manifiesto que sólo en un $20 \%$ de ellas se usan gafas plomadas y que en un $50 \%$ se usan guantes plomados (24). En la tabla I se exponen las dosis máximas permitidas de radiación absorbida por el personal sanitario (25).

Realizar la técnica eco-guiada permite al médico tener la certeza de que la punta de la aguja se sitúa en el espacio epidural a nivel sacro, de la misma manera que lo hace guiado mediante flouroscopia (14), aunque la mayor ventaja que ofrece esta técnica es la ausencia de radiaciones ionizantes y todo lo que estas conllevan $(13,25)$. Además, existen otras ventajas como la posibilidad transportar el ecógrafo o el no tener que utilizar contrastes yodados. Como desventaja cabe destacar que el uso del ecógrafo necesita de una curva de aprendizaje y una familiarización con la imagen ecográfica, además de la posibilidad de una inyección intravascular dentro del espacio epidural sacro que pasaría inadvertida al realizar la técnica con control ecográfico.

TABLA I. RECOMENDACIONES DE LA INTERNATIONAL COMMISSION ON RADIOLOGICAL PROTECTION (21)

Dosis máxima aceptable en exposición profesional por tejido (REM/año)

\begin{tabular}{ll}
\hline En cristalino & 15 mREM \\
Piel & 50 mREM \\
Manos y pies & $50 \mathrm{mREM}$ \\
\hline
\end{tabular}

\section{CONCLUSIÓN}

La realización del bloqueo caudal eco-guiado es una técnica fiable como alternativa a la técnica guiada con fluo- roscopia para asegurar la colocación correcta de la punta de la aguja en el espacio epidural, ofreciendo la ventaja de no ser una técnica nociva para el personal sanitario ni para el enfermo. Las complicaciones son poco frecuentes y cuando aparecen son de escasa gravedad.

\section{CORRESPONDENCIA:}

Agustín Mendiola de la Osa

Unidad del Dolor

Servicio Anestesiología, Reanimación y Terapéutica del Dolor Hospital General de Ciudad Real

C/ del Obispo Rafael Torija, s/n

13005 Ciudad Real

e-mail: ag_mendiola@hotmail.com

\section{BIBLIOGRAFÍA}

1. Botwin K, Brown LA, Fishman M, Rao S. Fluoroscopically guided caudal epidural steroid injections in degenerative lumbar spine stenosis. Pain Physician 2007;10:547-58.

2. Abdi S, Datta S, Trescot AM, Schultz DM, Adlaka R, Atluri SL, et al. Epidural steroids in the management of chronic spinal pain: A systematic review. Pain Physician 2007; 10:185-212.

3. Jöhr M, Berger TM. Caudal blocks. Paediatr Anaesth 2012;22(1):44-50.

4. Aggarwal A, Harjeet Sahni D. Morphometry of sacral hiatus and its clinical relevance in caudal epidural block. Surg Radiol Anat 2009;31:793-800.

5. Dadure C, Raux O, Rochette A, Capdevila X.Interest of ultrasonographic guidance in paediatric regional anaesthesia. Ann Fr Anesth Reanim 2009;28(10):878-84.

6. Manchikanti L, Singh V, Cash KA, Pampati V, Damron KS, Boswell MV. Effect of fluoroscopically guided caudal epidural steroid or local anesthetic injections in the treatment of lumbar disc herniation and radiculitis: A randomized, controlled, double blind trial with a two-year follow-up. Pain Physician 2012;15(4):273-86.

7. Manchikanti L, Cash KA, McManus CD, Pampati V, Fellows B Results of 2-year follow-up of a randomized, double-blind, controlled trial of fluoroscopic caudal epidural injections in central spinal stenosis. Pain Physician 2012;15(5):371-84.

8. Muntané Sánchez A, Fontes Caramé D, Mayoral Rojals V, Aja Rodríguez L. Aspectos técnicos en la infiltración caudal guiada por tomografía computarizada. Rev Soc Esp Dolor 2010;17(8):372-5.

9. Chen CP, Tang SF, Hsu TC, Tsai WC, Liu HP, Chen MJ, et al. Ultrasound guidance in caudal epidural needle placement. Anesthesiology 2004;101(1):181-4.

10. Yoon JS, Sim KH, Kim SJ, Kim WS, Koh SB, Kim BJ The feasibility of color Doppler ultrasonography for caudal epidural steroid injection. Pain 2005;118(1-2):210-4.

11. Domingo Rufes T, Mayoral V, Casals M, Serrano A, Miguel M, Sabaté A. La punción guiada con ultrasonidos aplicada a una unidad de dolor crónico. Rev Esp Anestesiol Reanim 2010;57:493-507.

12. Botwin KP, Thomas S, Gruber RD, Torres FM, Bouchlas CC, Rittenberg JJ, et al. Radiation exposure of the spinal interventionalist performing fluoroscopically guided lumbar 
transforaminal epidural steroid injections. Arch Phys Med Rehabil 2002;83(5):697-701.

13. Nota Técnica de Prevención 614: Radiaciones ionizantes: normas de protección. Ministerio de trabajo y asuntos sociales.

14. Chen CP, Tang SF, Hsu TC, Tsai WC, Liu HP, Chen MJ, et al. Ultrasound guidance in caudal epidural needle placement. Anesthesiology 2004;101(1):181-4.

15. Lundblad M, Eksborg S, Lönnqvist PA. Secondary spread of caudal block as assessed by ultrasonography. Br J Anaesth 2012;108(4):675-81.

16. Shin SK, Hong JY, Kim WO, Koo BN, Kim JE, Kil HK. Ultrasound evaluation of the sacral area and comparison of sacral interspinous and hiatal approach for caudal block in children. Anesthesiology 2009;111(5):1135-40.

17. Raghunathan K, Schwartz D, Connelly NR. Determining the accuracy of caudal needle placement in children: A comparison of the swoosh test and ultrasonography. Paediatr Anaesth 2008;18(7):606-12.

18. Arcelus Martínez JI. The new American College of Chest Physicians Antithrombotic Therapy and Prevention of Thrombosis Guidelines. Rev Esp Anestesiol Reanim 2012;59:287-8.

19. Llau JV. Anestesia regional y hematoma epidural: seguridad y trascendencia. Rev Soc Esp Dolor 2004;11(7):25-6.
20. Tsui BC, Tarkkila P, Gupta S, Kearney R. Confirmation of caudal needle placement using nerve stimulation. Anesthesiology 1999;91(2):374-8.

21. Stout A. Epidural steroid injections for low back pain. Phys Med Rehabil Clin N Am 2010;21(4):825-34.

22. Dashfield AK, Taylor MB, Cleaver JS, Farrow D. Comparison of caudal steroid epidural with targeted steroid placement during spinal endoscopy for chronic sciatica: A prospective, randomized, double-blind trial. Br J Anaesth 2005;94:514-9.

23. Manchikanti L, Pampati V, Rivera JJ, Beyer C, Damron KS, Barnhill RC. Caudal epidural injections with sarapin or steroids in chronic low back pain. Pain Physician 2001;4(4):322-35

24. Hernández García JM, Vidal Marcos A, Gasco García C. A survey on the use of fluoroscopy in the treatment of pain: Do we perform it correctly? Rev Esp Anestesiol Reanim 2012;59(8):430-5.

25. IRCP, 2007. The 2007 Recommendations of the international Commission on radiological protection. IRCP publication103. Ann. IRCP 37 (2-4). Disponible en: http://www. ircp.org/docs/P103_Spanish.pdf Consultado 7 Dic 2012$12-06$ 